\title{
Non-Markovian Regime Switching with Endogenous States and Time-Varying State Strengths
}

\author{
January 2004
}

\author{
Siddhartha Chib \\ Olin School of Business \\ Washington University \\ chib@olin.wustl.edu

\section{Michael Dueker*} \\ Federal Reserve Bank of St. Louis \\ P.O. Box 442, St. Louis, MO 63166 \\ mdueker@stls.frb.org; fax (314) 444-8731
}

* The content is the responsibility of the authors and does not represent official positions of the Federal Reserve Bank of St. Louis or the Federal Reserve System. 


\title{
Non-Markovian Regime Switching with Endogenous States and Time-Varying State Strengths
}

\begin{abstract}
This article presents a non-Markovian regime switching model in which the regime states depend on the sign of an autoregressive latent variable. The magnitude of the latent variable indexes the 'strength' of the state or how deeply the system is embedded in the current regime. The autoregressive nature of this non-Markovian regime switching implies time-varying state transition probabilities, even in the absence of an exogenous covariate. Furthermore, with time-varying regime strengths, the expected duration of a regime is time-varying. In this framework, it is natural to allow the autoregressive latent variable to be endogenous so that regimes are determined jointly with the observed data. We apply the model to GDP growth, as in Hamilton (1989), Albert and Chib (1993) and Filardo and Gordon (1998) to illustrate the relation of the regimes to NBER-dated recessions and the time-varying expected durations of regimes.
\end{abstract}

JEL classifications: F42, C25, C22

Key words: Regime switching, Markov Chain Monte Carlo 


\section{Introduction}

Autoregressive models are popular in economics because many economic variables appear to respond more to their own past values than they do to a distributed lag of any other variable. The same is likely true of regimes. If the conditions gradually become ripe for a regime change, it might not be possible to find an exogenous covariate whose evolution matches this ripening process. For example, when modeling the Volcker monetary policy regime change in 1979, a regime modeler might claim that the occurrence of high inflation engendered a shift in probability toward a new regime. If this were true, then a regime-switching model could include past inflation as an explanatory variable in Filardo's (1994) time-varying transition probability Markow switching model. In fact, however, the history of monthly or quarterly inflation rates (or almost any other extrinsic variable) does not presage a unique build-up of pressure for a change of monetary policy regime in 1979. Similarly, if the regime studied is the recession/expansion state of the business cycle, then a well-known problem is how to identify a variable or set of variables that indicates a coming shift from an expansion phase to a recession phase. In both of these cases, autoregressive dynamics might prove more useful than a distributed lag of any exogenous covariate in modeling a gradual shift in regime probabilties.

A related issue is the extent to which regimes are determined separately from the observable data. A negative shock that moves the business cycle phase toward the expansion state from the recession state is possibly associated with a negative shock to observed GDP growth. These two shocks do not have to be postively correlated, and they might even be negatively correlated, but regime modelers should be hesitant to assume that the regime is exogenous and uncorrelated with the innovations to the data affected by the regime. With our latent variable approach, it is straightforward to allow for endogenous regimes that are correlated with the observable data. Kim, Piger and Startz 
(2003) discuss classical maximum-likelihood estimation of a Markov switching model with endogenous regimes. As shown below, the adjustments needed for regime endogeneity are comparatively simple and straightforward in a Markov Chain Monte Carlo framework.

This article introduces a new non-Markovian regime switching model in which the regime states depend on the sign of an autoregressive latent variable. The magnitude of the latent variable indexes the 'strength' of the state or how deeply the system is embedded in the current regime. This non-Markovian regime switching automatically implies time-varying state transition probabilities. With autoregressive dynamics governing the transition probabilities, we can readily demonstrate how the expected duration of the current regime can vary across time. This model is readily contrasted with the typical two-state Markov switching model if we write the transition probabilities of a two-state Markov process as a function of a normally-distributed latent variable, $S^{*}$, that governs the binary regime indicator $S$ :

$$
\begin{aligned}
& S_{t}^{*}= \lambda+\theta S_{t-1}+e_{t} \\
& e_{t} \sim N(0,1) \\
& S_{t}=0 \Longleftrightarrow S_{t}^{*}<0
\end{aligned}
$$

The constant transition probabilities for this Markov process are therefore parameterized as

$$
\begin{aligned}
& P\left(S_{t}=0 \mid S_{t-1}=0\right)=\Phi(-\lambda) \\
& P\left(S_{t}=1 \mid S_{t-1}=1\right)=1-\Phi(-\lambda-\theta),
\end{aligned}
$$

where $\Phi($.$) is the cumulative standard normal density function. With constant transtition$ probabilities, the Markov switching model implies a constant expected duration of the current regime. 
The model we propose with autoregressive state strengths takes the form

$$
\begin{aligned}
S_{t}^{*}= & \lambda+\theta S_{t-1}^{*}+e_{t} \\
& e_{t} \sim N(0,1) \\
S_{t}= & 0 \Longleftrightarrow S_{t}^{*}<0
\end{aligned}
$$

This autoregressive latent variable generates a non-Markovian regime process because the probability of the state this period depends not only on the state last period but a continuous measure of the strength of the state last period. For these non-Markovian regimes, the time-varying state transition probabilities are

$$
\begin{aligned}
& P\left(S_{t}=0 \mid S_{t-1}^{*}\right)=\Phi\left(-\lambda-\theta S_{t-1}^{*}\right) \\
& P\left(S_{t}=1 \mid S_{t-1}^{*}\right)=1-\Phi\left(-\lambda-\theta S_{t-1}^{*}\right) .
\end{aligned}
$$

In the model with autoregressive state strengths, the probability of a regime change would rise if the latent index of regime strength, $S^{*}$, approached zero.

The Markov switching model time-varying transition probabilities introduced by Filardo (1994) would add lagged covariates, $Z$, such that

$$
\begin{aligned}
S_{t}^{*}= & \lambda+\theta S_{t-1}+\kappa Z_{t-1}+e_{t} \\
& e_{t} \sim N(0,1) \\
S_{t}= & 0 \Longleftrightarrow S_{t}^{*}<0 .
\end{aligned}
$$

Our model with autoregressive state strengths suggests that one strong candidate to be included in $Z_{t-1}$ is $S_{t-1}^{*}$, whereupon $S_{t-1}$ becomes unnecessary.

\section{MCMC estimation of non-Markovian regime switching}

We estimate the model with a latent autoregressive variable via Markov Chain Monte Carlo methods. MCMC methods for estimating the hidden Markov switching model of 
Hamilton (1989) were put forth in Albert and Chib (1993), who showed that once one augments the data with draws of the latent regime states, then the conditional distributions of the model parameters are often simple ordinary least squares distributions.

The specific model that we apply to GDP growth, denoted $y$, is

$$
\begin{aligned}
y_{t} & =\alpha_{1}+\left(\alpha_{0}-\alpha_{1}\right) I\left(S_{t}^{*}<0\right)+\phi y_{t-1}+u_{t} \\
S_{t}^{*} & =\lambda+\theta_{1} S_{t-1}^{*}+\theta_{2} y_{t-1}+e_{t}
\end{aligned}
$$

where $I($.$) is the indicator function and the covariance matrix of the error terms is$

$$
\operatorname{Cov}\left(\begin{array}{l}
u_{t} \\
e_{t}
\end{array}\right)=\Sigma=\left(\begin{array}{cc}
\sigma_{u}^{2} & \rho \\
\rho & 1
\end{array}\right) .
$$

The parameter groupings for MCMC estimation of the model are

$$
\begin{aligned}
& \varrho_{1}=\left(\lambda, \theta_{1}, \theta_{2}\right) \\
& \varrho_{2}=\left(\alpha_{0}, \alpha_{1}, \phi\right) \\
& \varrho_{3}=\Sigma \\
& \varrho_{4}=\left\{S_{t}^{*}\right\}, t=1, \ldots, T
\end{aligned}
$$

Conditional on $\left\{S_{t}^{*}\right\}$, the conditional distribution of the coefficients in $\varrho_{1}$ is Normal with the mean and variance implied by the Bayesian regression of $S_{t}^{*}$ on $y_{t-1}$ and $S_{t-1}^{*}$. With an uninformative prior, the conditional mean of $\varrho$ is simply the ordinary least squares estimator. To use regression techniques to derive a conditional mean and variance for $\varrho_{2}$, it is necessary to control for the endogeneity of $S^{*}$. Fortunately, the data augmentation makes this relatively simple. Conditional on $\left(\varrho_{1}, \varrho_{3}, \varrho_{4}\right)$, we can write

$$
u_{t}=\rho e_{t}+v_{t}
$$

where $v_{t}$ is uncorrelated with $e_{t}$, and re-write equation (6) as

$$
y_{t}-\rho e_{t}=\alpha_{0} I\left(S_{t}^{*}<0\right)+\alpha_{1} I\left(S_{t}^{*} \geq 0\right)+\phi y_{t-1}+v_{t}
$$


In this form, we have a regression equation in which the error term is uncorrelated with the regressor $I\left(S_{t}^{*}<0\right)$. This data-augmentation approach to endogenous regimes is considerably simpler than the classical approach outlined in Kim, Piger and Startz (2003). The priors used for these Bayesian regressions are discussed in the next section.

Conditional on $\varrho_{1}, \varrho_{2}, \varrho_{4}$, the residual series $\left\{u_{t}\right\}$ and $\left\{e_{t}\right\}$ are calculated and the approach from Chib, Greenberg and Jeliazkov (2003) is used to sample the covariance matrix $\Sigma$ using inverted Wishart distributions, subject to the restriction that $\Sigma_{2,2}=1$. A detailed discussion of sampling the autoregressive latent variable follows.

\section{Sampling the latent variable}

To reduce the degree of autocorrelation of the sampled values across MCMC iterations and to speed convergence of the sampler to the posterior distribution, multi-state sampling is preferable to single-state sampling of the latent variable. In single-state sampling, the conditional distribution of the latent variable this iteration would depend on values drawn for the previous iteration (with iteration number denoted as a superscript):

$$
f\left(S_{t}^{*(i+1)} \mid\left\{S_{j}^{*(i+1)}\right\}_{j<t},\left\{S_{k}^{*(i)}\right\}_{k>t},\left\{y_{t}\right\}\right)
$$

As suggested by Carter and Kohn (1994), Fruhwirth-Schnatter (1994) and de Jong and Shephard (1995), multi-state sampling can be carried out based on the identity

$$
f\left(\left\{S_{t}^{*}\right\} \mid\left\{y_{t}\right\}\right)=f\left(S_{T}^{*} \mid\left\{y_{t}\right\}\right) \prod_{t=1}^{T-1} f\left(S_{t}^{*} \mid S_{t+1}^{*},\left\{y_{j}\right\}_{j=1, . ., t}\right),
$$

using the Kalman filter to calculate the conditional distributions on the right side of eq. (11).

One complication is that the indicator function in eq. (6) makes it a nonlinear function of the latent variable, so we need to employ the extended Kalman filter [Welch and Bishop 
(2002)]. If we start with a canonical linear state-space model with observation variables $y$ and state variables $X$,

$$
\begin{aligned}
y & =H X_{t}+v_{t} \\
X_{t+1} & =F X_{t}+D Z_{t}+w_{t+1},
\end{aligned}
$$

then the well-known Kalman filtering equations are

$$
\begin{aligned}
X_{t+1 \mid t} & =F X_{t \mid t}+D Z_{t} \\
X_{t+1 \mid t+1} & =X_{t+1 \mid t}+K_{t+1}\left[y_{t+1}-H X_{t+1 \mid t}\right] \\
P_{t+1 \mid t} & =F P_{t \mid t} F^{\prime}+Q \\
P_{t+1 \mid t+1} & =P_{t+1 \mid t}-K_{t+1} H P_{t+1 \mid t} \\
K_{t+1} & =P_{t+1 \mid t} H^{\prime}\left(H P_{t+1 \mid t} H^{\prime}+R\right)^{-1}
\end{aligned}
$$

The non-Markovian regime switching model has the following state-space form:

$$
\begin{aligned}
y_{t} & =\alpha_{1}+\left(\alpha_{0}-\alpha_{1}\right) I\left(S_{t}^{*}<0\right)+\rho e_{t}+v_{t} \\
\left(\begin{array}{c}
e_{t+1} \\
S_{t}^{*}
\end{array}\right) & =\left(\begin{array}{cc}
0 & 0 \\
1 & \theta_{1}
\end{array}\right)\left(\begin{array}{c}
e_{t} \\
S_{t-1}^{*}
\end{array}\right)+\left(\begin{array}{cc}
0 & 0 \\
\lambda & \theta_{2}
\end{array}\right)\left(\begin{array}{c}
1 \\
y_{t-1}
\end{array}\right)+\left(\begin{array}{c}
e_{t+1} \\
0
\end{array}\right)
\end{aligned}
$$

The extended Kalman filter takes Jacobians of nonlinear functions to arrive at updating matrices for the Kalman filtering equations. Extended Kalman filtering consists of taking a Taylor series linearization around the expected values of the arguments of the nonlinear function. Here we define $G\left(X_{t}\right)=I\left(X_{t}\right)-P\left(X_{t} \mid I_{t-1}\right)$. The Taylor series 
approximation around the expected value $X_{0 t}=X_{t \mid t-1}=\left(w_{t}=0, S_{t-1}^{*}=S_{t-1 \mid t-1}^{*}\right)$ leads to

$I\left(S^{*}\left(X_{t}\right)<0\right) \approx P\left(S^{*}\left(X_{t}\right)<0 \mid I_{t-1}\right)+I\left(S^{*}\left(X_{0 t}\right)<0\right)-P\left(S^{*}\left(X_{0 t}\right)<0 \mid I_{t-1}\right)+H_{t}\left(X_{t}-X_{0 t}\right)$,

where $H_{t}$ is the Jacobian of $G\left(X_{t}\right)$ evaluated at $X_{0 t}$. At $X_{0 t}, P\left(S^{*}\left(X_{0 t}\right)<0 \mid I_{t-1}\right)$ takes on either the value zero or one and is equal to $I\left(S^{*}\left(X_{0 t}\right)<0\right)$, so these two terms cancel.

In this example, the $1 \times 2$ Jacobian vector includes a finite-difference approximation to differentiating the indicator function:

$$
H_{t}=\frac{\partial G}{\partial X} \approx \frac{E \Delta I\left(S^{*}\left(X_{t}\right)<0\right)}{E\left[\xi_{t} \mid S_{t}^{*}<0, S_{t-1}^{*}<0\right]} \frac{\partial \xi_{t}}{\partial X_{t}}-\frac{\partial P\left(S^{*}\left(X_{t}\right)<0 \mid I_{t-1}\right)}{\partial \xi_{t}} \frac{\partial \xi_{t}}{\partial X_{t}}
$$

where

$$
\xi_{t}=\frac{S_{t}^{*}-\lambda-\theta_{1} S_{t-1 \mid t-1}^{*}-\theta_{2} y_{t-1}}{\left(P_{t \mid t-1}^{(1,1)}-2 \theta_{1} P_{t \mid t-1}^{(1,2)}+\theta_{1}^{2} P_{t \mid t-1}^{(2,2)}\right)^{0.5}}
$$

and superscript $(2,2)$ indicates the element of the matrix. Because $\xi_{t}$ is a standard normal,

$$
\begin{aligned}
E\left[\xi_{t} \mid S_{t}^{*}>0\right]= & \phi\left(\xi_{t} \mid S_{t}^{*}=0\right) /\left[1-\Phi\left(\xi_{t} \mid S_{t}^{*}=0\right)\right] \\
E\left[\xi_{t} \mid S_{t}^{*}<0\right]= & -\phi\left(\xi_{t} \mid S_{t}^{*}=0\right) / \Phi\left(\xi_{t} \mid S_{t}^{*}=0\right) \\
\frac{E \Delta I\left(S^{*}\left(X_{t}\right)<0\right)}{E\left[\xi_{t} \mid S_{t}^{*>}<, S_{t-1}^{*}<0\right]}= & -1\left[1-\Phi\left(\xi_{t} \mid S_{t}^{*}=0\right)\right] I\left(S_{t-1}^{*}<0\right) / E\left[\xi_{t} \mid S_{t}^{*}>0\right] \\
& +\Phi\left(\xi_{t} \mid S_{t}^{*}=0\right) I\left(S_{t-1}^{*}>0\right) / E\left[\xi_{t} \mid S_{t}^{*}<0\right] \\
\frac{\partial \xi_{t}}{\partial X_{t}}= & \left(\begin{array}{c}
1 \\
\theta_{1}
\end{array}\right)^{\prime} /\left(P_{t \mid t-1}^{(1,1)}-2 \theta_{1} P_{t \mid t-1}^{(1,2)}+\theta_{1}^{2} P_{t \mid t-1}^{(2,2)}\right)^{0.5}
\end{aligned}
$$

where $\phi($.$) is the standard normal density function and \Phi($.$) is the cumulative standard$ normal density. The ratio of finite differences in $H_{t}$ represents the probability that a shock to the latent variable will cause a change in the indicator function times the sign of the change in the indicator function divided by the expected value of the shock conditional on it being large enough to induce a regime change. 
The extended Kalman filtering equations are altered from the canonical form of eq. (14) to

$$
\begin{aligned}
X_{t+1 \mid t} & =F X_{t \mid t} \\
X_{t+1 \mid t+1} & =X_{t+1 \mid t}+K_{t+1}\left[y_{t+1}-\phi y_{t-1}-\alpha_{1}-\left(\alpha_{0}-\alpha_{1}\right) P\left(S^{*}\left(X_{t+1}\right)<0 \mid I_{t}\right)\right] \\
P_{t+1 \mid t} & =F P_{t \mid t} F^{\prime}+Q \\
P_{t+1 \mid t+1} & =P_{t+1 \mid t}-K_{t+1} H_{t+1} P_{t+1 \mid t} \\
K_{t+1} & =P_{t+1 \mid t} H_{t+1}^{\prime}\left(H_{t+1} P_{t+1 \mid t} H_{t+1}^{\prime}+R\right)^{-1}
\end{aligned}
$$

where

$$
P\left(S^{*}\left(X_{t+1}\right)<0 \mid I_{t}\right)=\Phi\left(\xi_{t+1} \mid S_{t+1}^{*}=0\right) .
$$

We also need to apply one smoothing step following the sampling of $X_{t+1}$ :

$$
\begin{aligned}
& X_{t \mid t+1}=X_{t \mid t}+P_{t \mid t} F^{\prime} P_{t+1 \mid t}^{-1}\left(X_{t+1}-X_{t+1 \mid t}\right) \\
& P_{t \mid t+1}=P_{t \mid t}+P_{t \mid t} F^{\prime} P_{t+1 \mid t}^{-1}\left(P_{t+1 \mid t+1}-P_{t+1 \mid t}\right)\left(P_{t \mid t} F^{\prime} P_{t+1 \mid t}^{-1}\right)^{\prime}
\end{aligned}
$$

In this way, the latent variable is sampled in reverse order, starting with $X_{T}$.

Because the extended Kalman filter only provides approximately Gausian errors, the values of the latent variable drawn this way are only considered candidate values. Without an exact conditiional distribution, we subject these candidate values to a MetropolisHastings step where a candidate draw can be rejected.

\section{Application to business cycle phases}

In applying this regime switching model to GDP growth, we found that an informative prior is necessary to slow down the fluctuations in the latent variable. With an uninformative prior, the inferred latent $S^{*}$ series closely mimics the data $y$ with a different variance. 
The estimated values of growth states, $\alpha_{0}$ and $\alpha_{1}$, are also closer together than one would associate with two distinct business cycle regimes in the absence of an informative prior. In the Bayesian regression, as shown in Chib and Greenberg (1996), the coefficients are normally distributed such that

$$
\begin{aligned}
\beta \sim & N\left(\hat{\beta}, B_{n}^{-1}\right) \\
& B_{n}=B_{0}+X^{\prime} X / \sigma^{2} \\
& \hat{\beta}=B_{n}^{-1}\left(B_{0} \beta_{0}+X^{\prime} y / \sigma^{2}\right),
\end{aligned}
$$

where $X$ is set of regressors, $y$ is the regressand, $\sigma^{2}$ is the variance and, most importantly, $B_{0}$ is a diagonal matrix that determines the strength of the prior placed on the set of coefficient values $\beta_{0}$. For the GDP growth regression of eq. (10), where the coefficients are $\left(\alpha_{0}, \alpha_{1}, \phi\right)$, the diagonal elements of $B_{0}$ were set to $(300,300,0)$ and $\beta_{0}$ was set to $(0.10,0,80,0.10)$, so that no prior was placed on the lagged dependent variable. For the latent state from eq. (6), where the coefficients are $\left(\lambda, \theta_{1}, \theta_{2}\right)$, the diagonal elements of $B_{0}$ were set to $(0,100,0)$ and $\beta_{0}$ was set to $(0.10,0.80,0.10)$, so that the prior only served to lift the autoregressive coefficient, $\theta_{1}$. Experimentation showed that these priors were strong enough to prevent the regime from changing in more than one-third of the observations; when the regime changes more often than this, the model is trying to fit high-frequency fluctuations between two expansionary growth states, as opposed to lowerfrequency business cycle fluctuations.

One obvious question is why the Markov switching regimes of Hamilton (1989) do not require any prior restrictions in order to match business cycle fluctuations, whereas the present non-Markovian regime switching model does. Consider first the Markov switching model. Suppose that it tried to fit high-frequency fluctuations between two expansionary growth states of 2.5 and 4 percent annualized growth. With fixed transition probabilities 
the model would need to have states that were not very persistent to have relatively frequent transitions. As a consequence though, the one-step-ahead forecast of output growth would not vary much across time, so little would be gained in terms of the likelihood function value. Consider, in contrast, the non-Markovian regime switching from eq. (3), in which transition probabilities automatically are time-varying. In this model one can have both frequent regime transitions and one-step-ahead forecasts of output growth that vary considerably across time. All it takes in eq. (3) is for the unconditional mean of the latent variable, $S^{*}$, to be near zero and for the autoregressive coefficient, $\theta$, to be greater than zero. Then, the conditional mean of $S^{*}$ can differ from zero, causing the one-step-ahead forecasts of output growth to differ from the unconditional mean.

For the model with the informative priors discussed above, the MCMC sampler was run through 6500 iterations with the first 1500 iterations discarded to allow the sampler to converge on the posterior distribution. Quarterly GDP growth from 1960Q1 to 2003Q4 was used to estimate the non-Markovian regime switching model. Table 1 shows the posterior means and 90 percent probability intervals for the coefficients. 


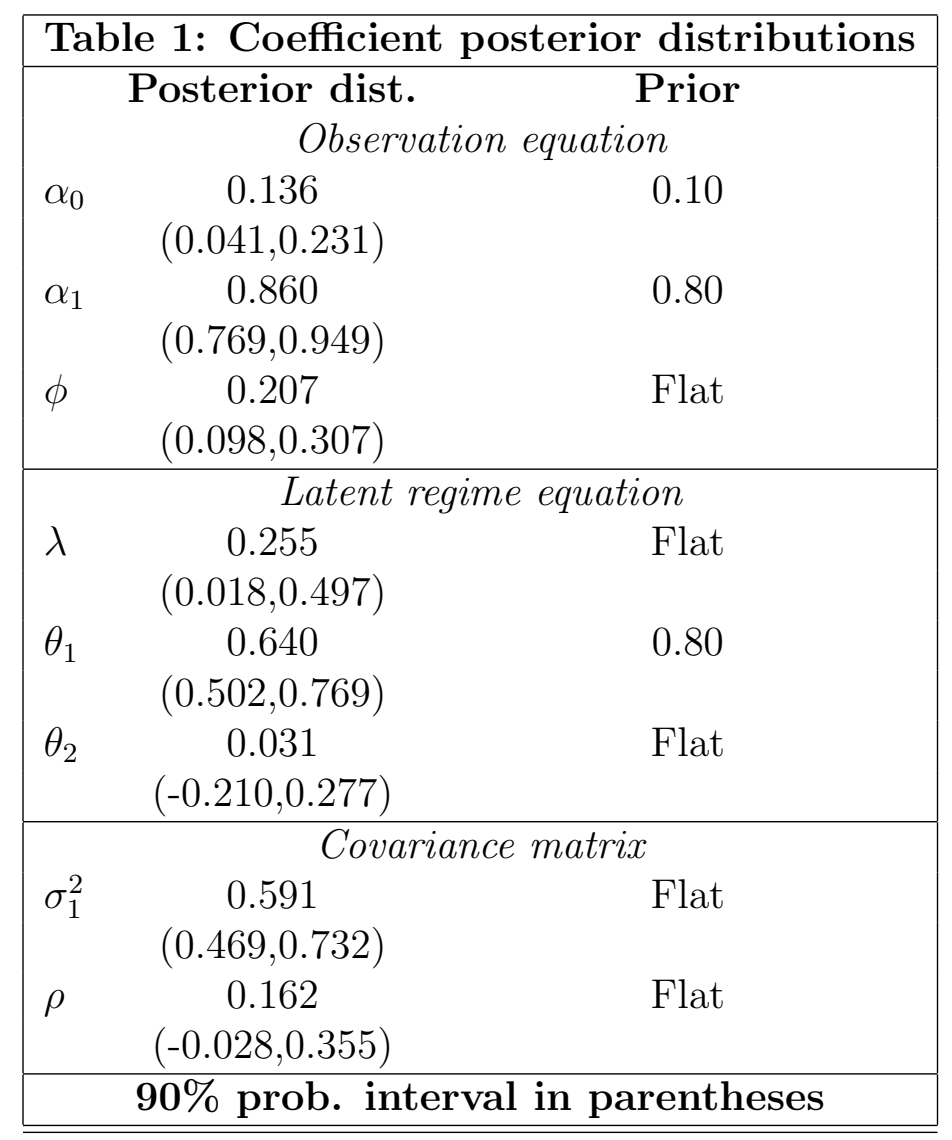

The posterior means of the $\alpha$ intercepts in the observation equation move less from the prior values than does the autoregressive coefficient, $\theta_{1}$, in the latent regime equation. The fact that the model finds that the autoregressive coefficient, $\theta_{1}$, is centered far from zero in the latent regime equation supports the idea that the index of regime strength, $S^{*}$, responds more closely to its own past value than to other variables, such as $y_{t-1}$.

\section{Matching NBER business cycle turning points}

The most interesting output from the non-Markovian regime switching model of GDP growth is the posterior mean of the latent, strength-of-regime indicator, $S^{*}$. Figure 1 
shows this posterior mean and how well its crossings of zero match the NBER business cycle turning points. The biggest discrepancy between the sign of the posterior mean of the latent variable and the NBER recession dates occurs at the March 1991 NBER trough date. The posterior mean of the latent variable remained below zero through the fourth quarter of 1991. In all, the regime-switching model had a posterior mean below zero for six quarters in 1990-91, whereas the NBER recession lasted only three quarters. In 2001, in contrast, the posterior mean was below zero for four quarters, starting in the first quarter of 2001, whereas the NBER recession lasted three quarters, starting with the second quarter of 2001. Overall, however, the regime switching model implies switching dates that are very close to NBER turning points throughout the sample. The posterior mean of the latent variable can also serve as a business cycle index, given that it measures the strength of growth rate regimes. For example, 1972, 1978 and 1984 are periods of pronounced cyclical strength. Similarly, the milder recessions are reflected in the posterior mean of the latent variable as recessions where the the latent variable did not dip as far below zero, such as in 1970 and 2001. We also calculate posterior means of the regime probabilities, calculated as the percentage of the 5000 draws that the latent variable, $S_{t}^{*}$, was above zero. For this measure, a posterior mean probability of 0.5 corresponds closely with NBER turning points, as shown in Figure 2. To reiterate, the priors needed to induce the latent variable to change signs at the business cycle frequency included a prior to keep the two growth rate parameters, $\alpha_{0}$ and $\alpha_{1}$, sufficiently far apart so as not to reflect fast and moderate growth within economic expansions; the other prior was to ensure a degree of persistence on the latent variable by way of the autoregressive coefficient, $\theta_{1}$.

\section{Time-varying expected regime durations}


With positive serial dependence, the farther the autoregressive latent variable is from zero (the greater the strength of the current regime), the higher is the expected time before a sign (regime) change. Here we illustrate this feature of the non-Markovian regime switching model with calculations of time-varying expected durations. Starting with the posterior mean value of the latent variable, $S_{t}^{*}$, and posterior mean values of the parameters, we simulated shock processes for eq. (6) until the sign changed at $S_{t+k}^{*}$, where $k$ is the duration of the regime from time $t$. Note that if we started from the value of $S_{t}^{*(i)}$ from each iteration $i$ of the MCMC sampler, we would be mixing cases where $S_{t}^{*(i)}$ was positive and negative. For this reason, we use the posterior mean values as a common starting point. The mean value of $k$ from 5000 simulations was calculated as the expected duration. Figure 3 plots these expected durations. Given the positive intercept, $\lambda$, the expected durations are longer on average when $S_{t}^{*}$ is above zero (for expansions) than when it is below zero (for recessions). On average, the expected duration in the expansion regime is about three times as long as in the recession regime. This ratio suggests that about 25 percent of the observations will pertain to the recession regime and this figure is not much different from the 21 percent of quarters that the NBER has declared to be recessions. Of course, the expected durations presented here are only in-sample estimates for the purpose of illustrating this feature of the model.

\section{A Monte Carlo investigation of the estimation procedure}

To investigate how well the extended Kalman filter uncovers the parameters of the data-generating process for the latent variable, we performed a Monte Carlo simulation. We generated 800 samples of artificial data, each with 200 observations, based on eqs. 
(6) and (7). The parameter values used were close to those estimated from the GDP growth data in Table 1. For each sample, we ran the MCMC estimation procedure with 6500 iterations and we saved the posterior means of the parameter draws from the last 5000 iterations. We then calculated the mean of the ensemble of 800 sets of posterior means to see how closely it conformed with the true values used to generate the data. Table 2 shows this result from the Monte Carlo investigation. As one might expect, the MCMC estimation procedure does quite well in recovering the parameter values for the observation equation. In the latent regime equation, there is some downward bias on the autoregressive parameter, $\theta_{1}$, probably in the form of an errors-in-variable bias. Additionally, the estimated correlation between the error terms in the observation and latent regime equations is biased downward somewhat. Overall, however, the MCMC estimation procedure with data augmentation recovers the salient features of the artificial data.

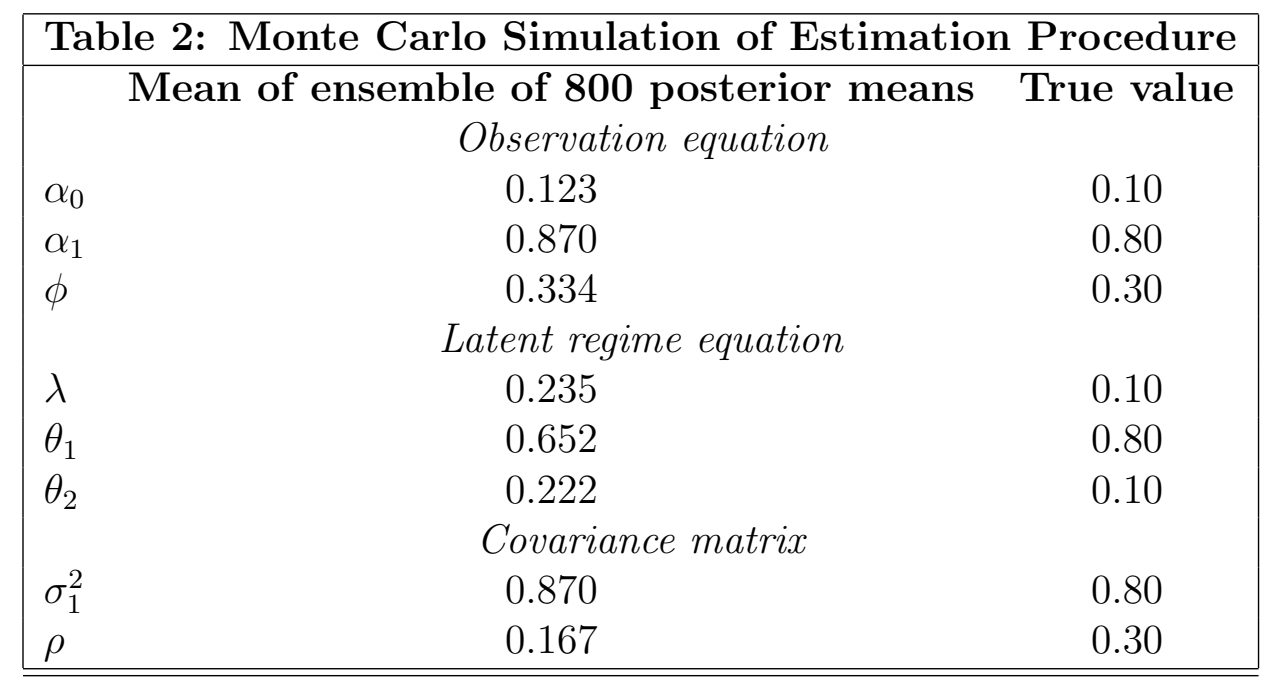

\section{Conclusions}


In this article, we present a non-Markovian regime switching model in which the magnitude of the latent variable indexes the strength of the state. In our application to regime switching at the business cycle frequency in the growth rate of GDP, we find that the posterior mean of the latent variable looks much like a business cycle index that indicates the degree of cyclical strength or weakness in the economy. Another useful feature of the non-Markovian model is the time-varying nature of its transition probabilities. Within this framework, it is straightforward to calculate the expected duration of the current regime for each observation.

We also demonstrate the straightforward adjustment one can make within the MCMC estimation procedure to allow for the regime process to be correlated with the observable data. Our estimates of GDP growth indicate that the regimes are not determined independently from the observed data. Instead, our monte carlo simulation suggests that our estimates of the correlation might be downwardly biased and might understate, if anything, the true magnitude of this correlation and the endgoeneity of the regimes. 


\section{REFERENCES (incomplete)}

Hamilton, J. D. (1989), "A New Approach to the Economic Analysis of Nonstationary Time Series and the Business Cycle," Econometrica 57, iss. 2, 357-84.

Albert, J. H. and Chib, S. (1993), "Bayes Inference via Gibbs Sampling of Autoregressive Time Series, Subject to Markov Mean and Variance Shifts," Journal of Business and Economic Statistics 11, iss. 1, 1-15.

Filardo, A. J. and Gordon, S. F. (1998), "Business Cycle Durations," Journal of Econometrics 85, iss. 1, 99-123.

Filardo, A. J. (1994), "Business-Cycle Phases and Their Transitional Dynamics," Journal of Business and Economic Statistics 12, iss. 3, 299-308.

By Chib, S. and Greenberg, E. (1996), "Markov Chain Monte Carlo Simulation Methods in Econometrics," Econometric Theory 12, iss. 3, 409-31. 


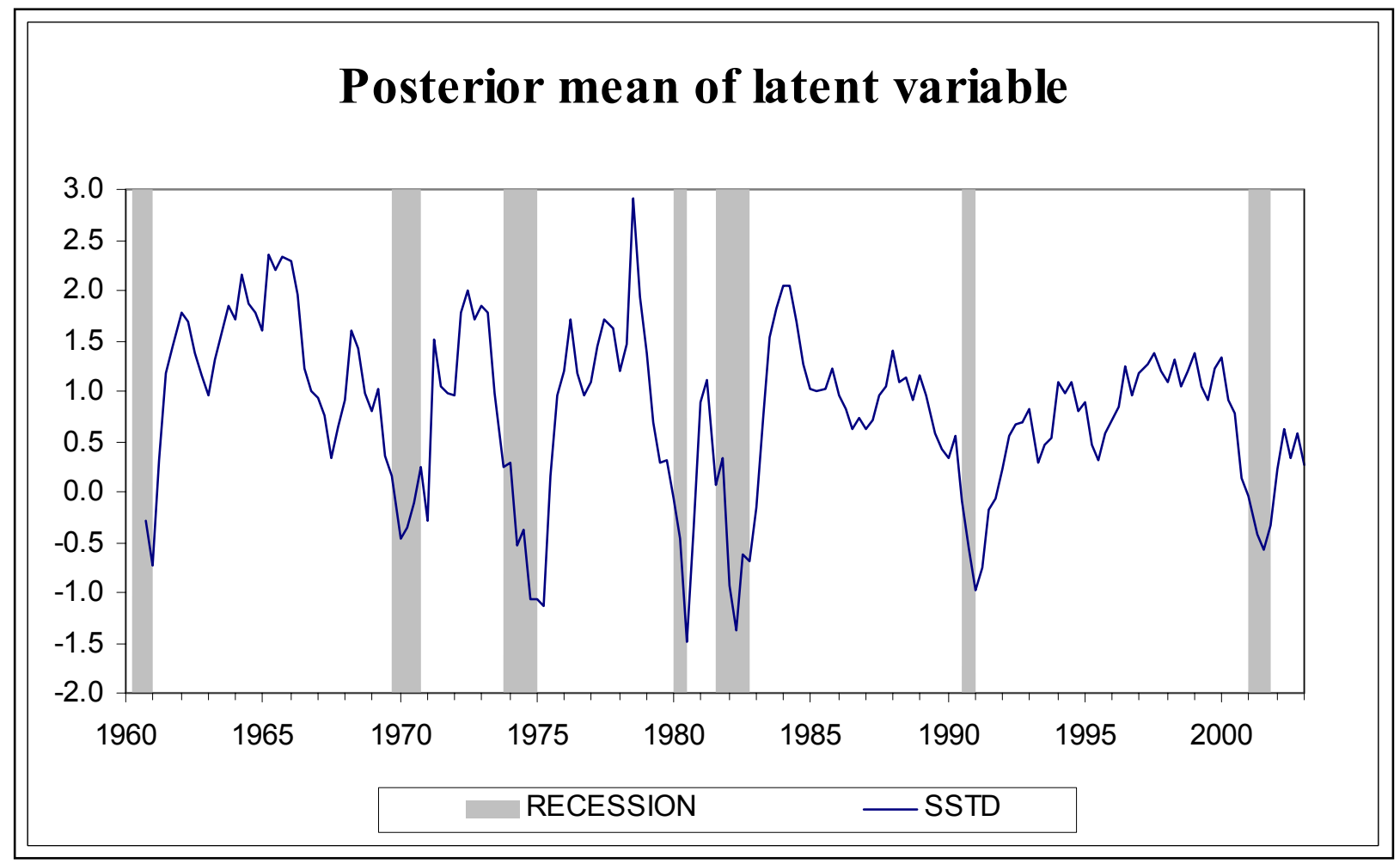




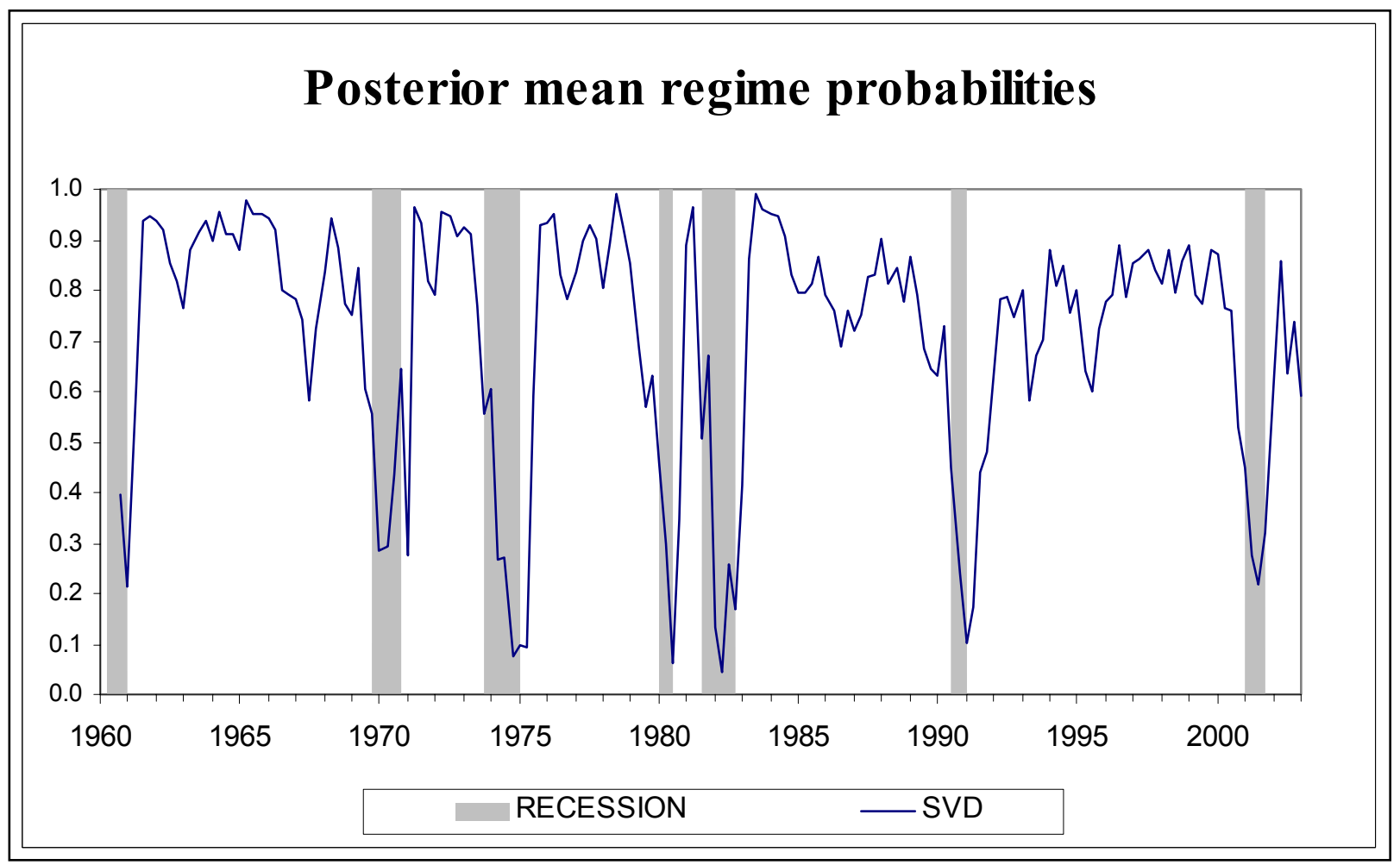


Time-Varying Expected Duration of Regime

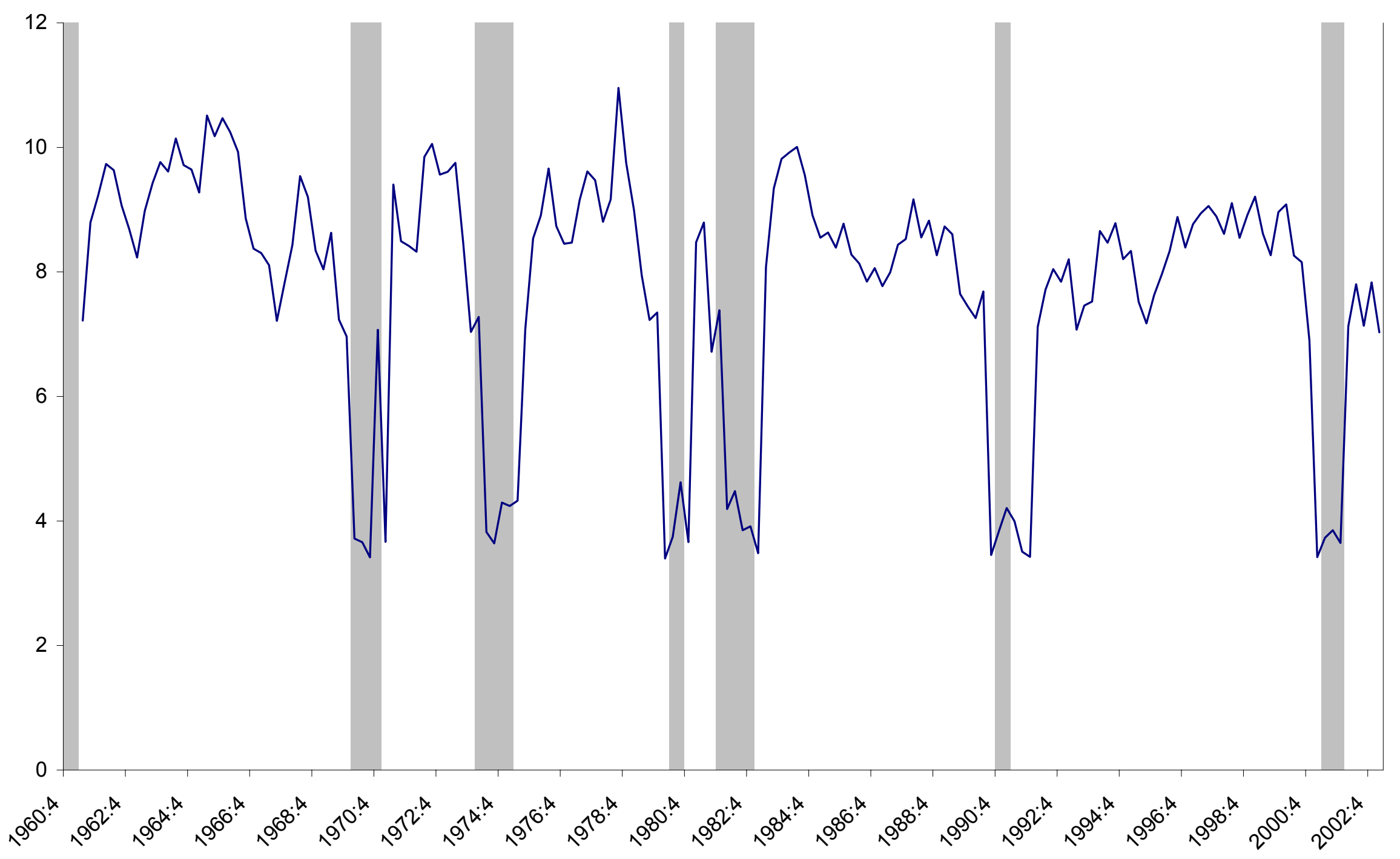

